\title{
EVOLUTION OF LASER LIGHT INDUCED SODIUM CLUSTERS
}

\author{
M. Kolwas, K. Kolwas and D. JakubczyK \\ Institute of Physics, Polish Academy of Sciences \\ Al. Lotników 32/46, 02-668 Warszawa, Poland
}

\begin{abstract}
We investigated the growing sodium clusters, induced by the laser light resonant with a sodium dimer transition. We observed the intensity of light scattered by clusters. The clusters were produced in the stainless steel cell containing sodium vapor and helium. We obtained data on cluster mean size and dynamics from fitting the theoretical formulas to the experimental data. The model of clusters' evolution basing on the thermodynamics of the vapor-liquid transition was applied.
\end{abstract}

PACS numbers: $36.40 .+\mathrm{d}, 31.70 . \mathrm{Hq}, 64.70 .-\mathrm{p}$

\section{Introduction}

Over the liquid metal surface there exists metal vapor in thermodynamic equilibrium with the liquid. One can find atoms, diatomic molecules, and molecules consisting of three or more atoms in the vapor. These multiatomic "molecules" are called clusters. Under the ordinary equilibrium thermodynamic conditions clusters are rare. Still there are methods that can provide us with relatively high concentrations of clusters. All the methods of cluster production are based on the supersaturating of vapor. The supersaturation pushes the system out of the equilibrium. Condensation in such a system leads to the formation of small spheres. In our case these are metal droplets.

The supersaturation may be obtained, for instance, as a result of expansion of the vapor mixed with the inert gas into the vacuum through a nozzle. It might be achieved as well by expanding of the vapor into the inert gas [1]. Another way is shown in Ref. [2] - the supersaturation was obtained by "pushing" the hot vapor into the colder zones with the aid of the laser beam.

In the experiment we conducted [3] clusters were induced by the laser light interacting with the sodium vapor. The process was taking place in a steel cell containing the sodium vapor and the buffer gas (helium under the pressure close to the atmospheric). Under certain conditions, defined by the temperature of the oven, the pressure of helium and the $\mathrm{Ar}^{+}$laser beam power, the laser light causes condensation and produces clusters. 
We investigated the process of formation of clusters by observing the intensity of light scattered by clusters. To describe the evolution of the observed light intensity, we considered a few methods of description of the light scattering and chose the modified Rayleigh-Guns formula as satisfactory for our problem [4]. Then we proposed a model describing the evolution of clusters starting from the nucleation (the moment of the inducing laser light switch-on) and ending at the thermodynamic equilibrium (stationary droplet size reached). We showed suitable calculations.

The proposed model enabled us to write a complete formula, which we fitted numerically to the experimental results. The analysis of this fit led us to the following conclusions:

- the rate of growth of clusters is time independent for broad range of parameters,

- this rate of growth is an increasing function of the laser light power, temperature of the oven and the buffer gas pressure,

- the equilibrium cluster size is a decreasing function of the laser light power and an increasing function of the buffer gas prcssurc and the temperature of the oven, and it tends to about $0.45 \mu \mathrm{m}$.

\section{Clusters in the cell}

Our cluster producing experiments were conducted in the stainless steel cell (ca. $30 \mathrm{~cm}$ long, $3 \mathrm{~cm}$ in diameter), a Ileat Pipe Oven working out of IIPO regime under about 500 torr pressure of helium. After warm-up we obtain saturated vapor at the temperature of about $750 \mathrm{~K}$ and then we switch on the $\mathrm{Ar}^{+}$laser working at $488 \mathrm{~nm}$ and resonantly exciting $\mathrm{Na}_{2}$ molecules. At the beginning only the greenish dimer fluorescence along the lascr beam passing the cell is visible. After a few seconds one can observe the appearance of the blue haze. Then the bright blue spots diversify from the blue mist and slowly start to dominate. Finally only the spots are visible on the blue background. The blue haze consists of small clusters, which scatter the light elastically. We can observe them as separate spots when their size approaches a fraction of a $\mu \mathrm{m}$.

In Fig. 1 an experimental setup that enables us to do measurements of scattered light intensity as a function of time is shown. A sample set of experimentally obtained dependencies for various cell temperatures is shown in Fig. 2. For relatively low temperatures (for instance $475^{\circ} \mathrm{C}-$ curve d) the intensity of the scattered light increases monotonously until it reaches maximum.

In Fig. 3 the examples of experimentally obtained dependencies for various buffer gas pressures are shown. For 650 torr the system passes from the state of the absence of clusters to the equilibrium state without the process of intense light scattering (manifesting as a well-pronounced maximum). Instead of that the very clear oscillation structure appears. As the buffer gas pressure decreases the maximum grows up and the frequency of oscillations decreases.

In order to describe the observed phenomenon we have to answer a few questions: 


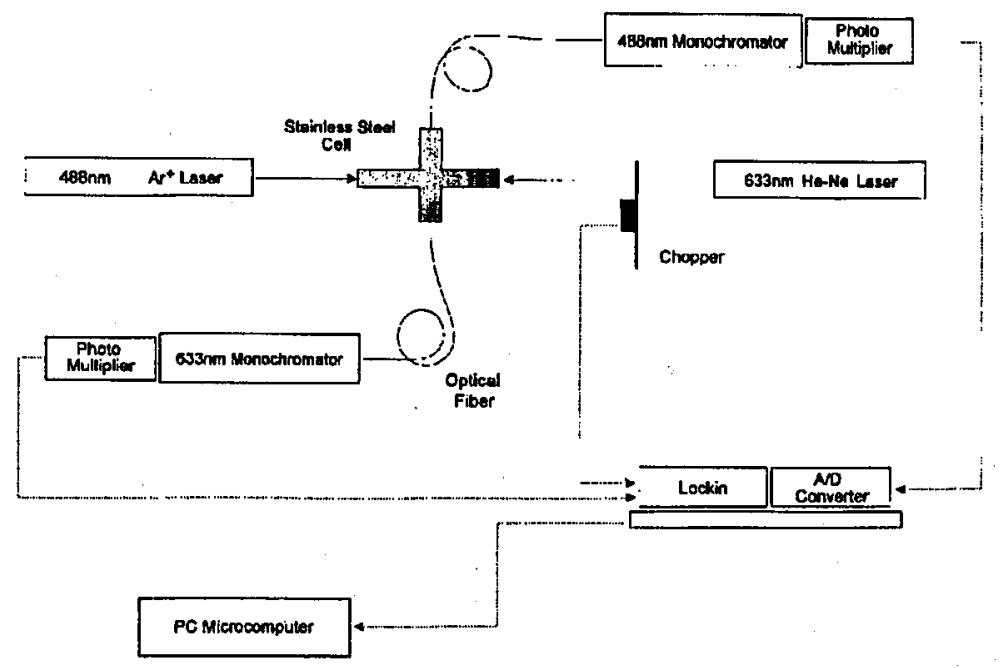

Fig. 1. The basic experimental setup.

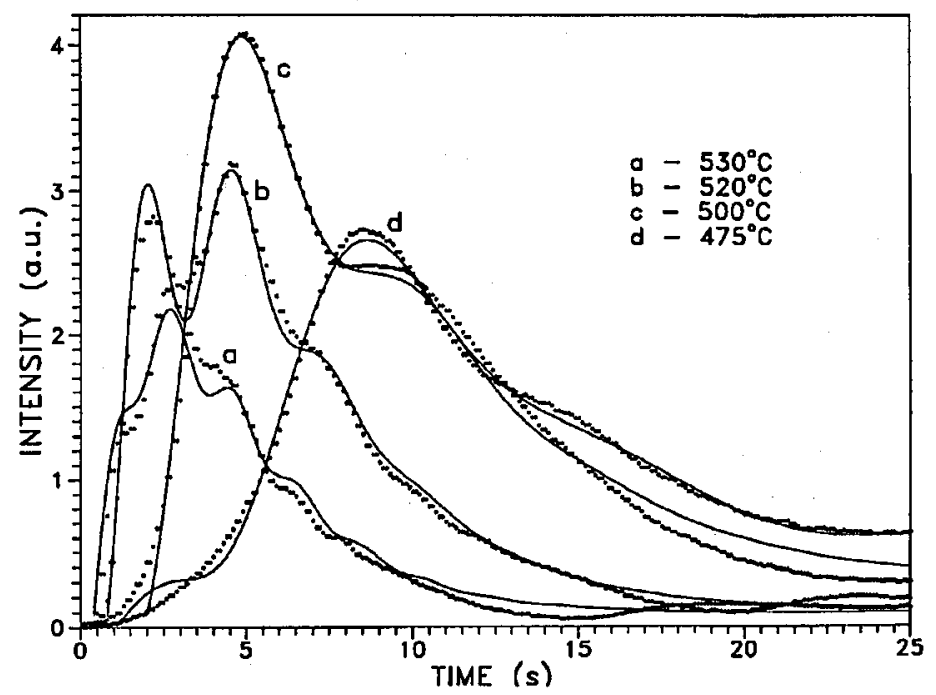

Fig. 2. The evolution of clusters for various temperatures of the cell.

1. What is the immediate cause of the condensation, or other words, why the sodium vapor supersaturation appears after the laser light switch-on?

2. How should we describe the scattering of light?

3. Do all the clusters have the same radius, or at least the well-defined average radius?

We tried to answer these questions one by one. 

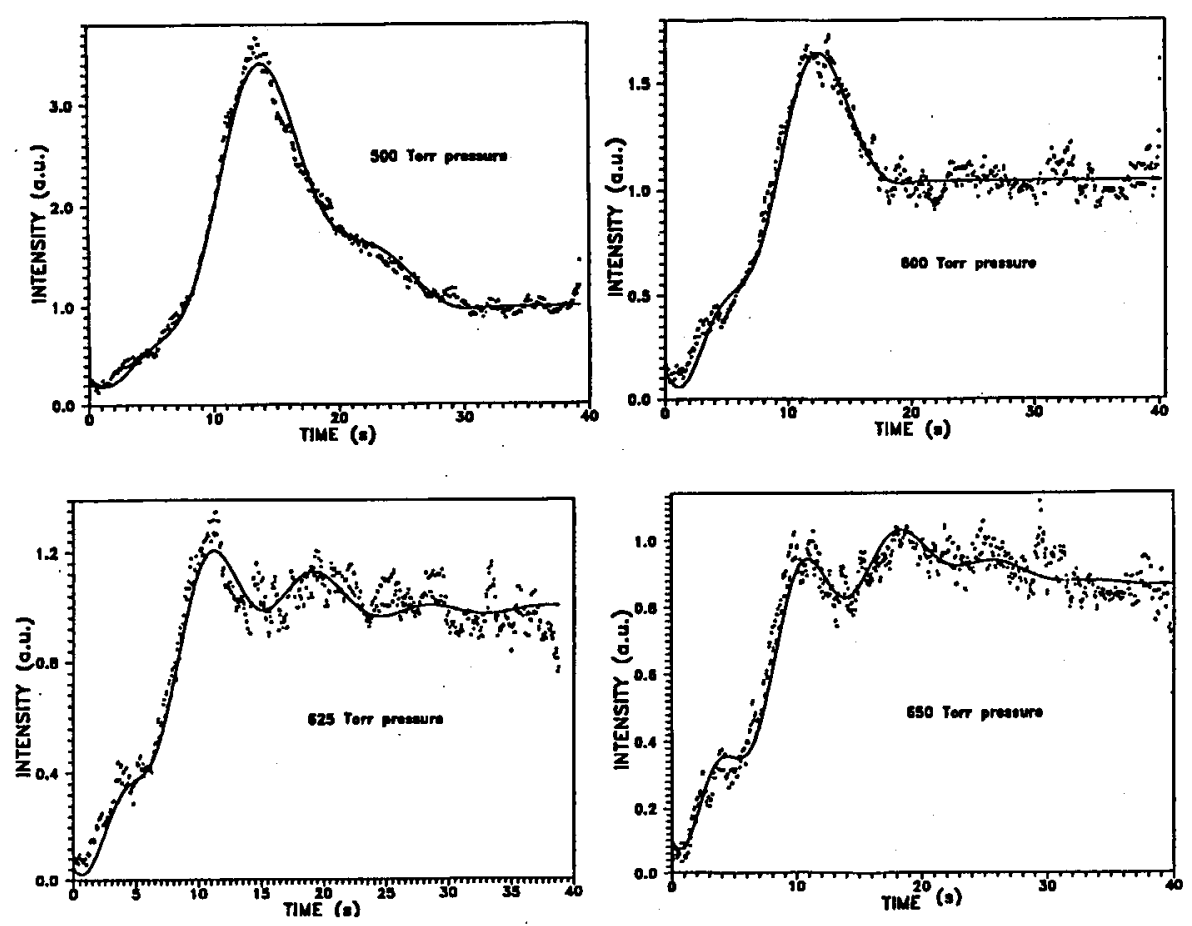

Fig. 3. The evolution of clusters for various buffer gas pressures.

\section{The formation and the evolution of clusters}

As it has already been mentioned the supersaturation of vapor is essential for the condensation and aggregation of clusters. The necessary excess of atoms in comparison with the thermodynamic equilibrium concentrations can be simply achieved by sudden cooling of vapor.

In our case the condensation takes place as a result of the laser light turn-on. The phenomenon manifests only when the laser light is in the resonance with the diatomic sodium molecules and when the bufter gas (helium) pressure is sufficiently high. It implies that the main source of non-equilibrium is the collision induced (with the atoms of helium) dissociation of the $\mathrm{Na}_{2}$ molecules excited with the laser light. There are two channels for such a dissociation. One is the dissociation of the $B^{1} \Pi_{u}$ state leading to the decay of the molecule into one ground state atom and one excited ${ }^{3} P$ atom. The excited atom emits yellow sodium light that was observed in the experiment. Another way is the collision transition of the excited molecule into the triplet state, for instance $A^{3} \Pi_{u}$, followed by the IR transition. The lowest triplet state ol sodium is very weakly bound and it dissociates into two sodium atoms. Both ways lead to the production of sodium atoms. So the laser light produces the excess of the sodium atoms indirectly by the collision induced dissociation of resonantly excited $\mathrm{Na}_{2}$ molecules. 
The formation of clusters-droplets of liquid sodium from the supersaturated vapor is a phase transition. The total free enthalpy (Gibbs function) of the system consisting of a separate droplet of the liquid and the bulk liquid metal is

$$
G_{\text {total }}^{\text {single }}=s M_{\mathrm{v}} g_{\mathrm{v}}+M_{1} g_{1}+M_{\mathrm{d}} g_{1}+4 \pi \gamma r^{2},
$$

where $s M_{\mathrm{v}}, M_{1}$ and $M_{\mathrm{d}}$ are the masses of the supersaturated vapor, bulk liquid and liquid in the droplet, respectively; $g_{\mathrm{v}}$ and $g_{1}$ are free enthalpies per mass unit of the vapor and liquid, respectively. $\gamma$ is the surface tension of liquid sodium in helium. While $s$ is the supersaturation and changes during the process of condensation, $M_{\mathrm{v}}$ is the mass of saturated vapor and remains constant as long as temperature does not change. $G_{\text {total }}^{\text {single }}$ is conscrved during the process so $\delta G_{\text {total }}^{\text {single }}=0$.

This leads to the condition for the radius of the droplet in the equilibrium with the vapor [5]

$$
P_{r}(T)=P_{\infty}(T) \exp \left(\frac{2 m \gamma}{\rho r k T}\right),
$$

where $m$ is atomic mass of helium, $\rho$ is the density of the droplet, $P_{r}$ and $P_{\infty}$ are the vapor pressures over the droplet surface and flat surface, respectively.

If we define the supersaturation as $s=P_{r}(T) / P_{\infty}(T)(\geq 1)$ we get the radius for the stable droplet in thermodynamic equilibrium with the environment

$$
r=\frac{2 \gamma}{\rho k T\left(\ln P_{r}-\ln P_{\infty}\right)}=\frac{2 \gamma}{\rho k T \ln (s)} .
$$

One has to remember that

$$
\ln P_{\infty}=a-\frac{b}{T}-c T+\ln T
$$

where $a, b, c$ and $d$ constants are tabularized [6].

The laser light produces the definite supersaturation and sodium droplets of the defined size. It is worth noticing that the increase in the light intensity leads to the increase in the supersaturation and to the decrease in the stable cluster radius (Eq. (3)).

All the calculations considering enthalpy can be repeated for the set of $N$ droplets, each of the radius $r$, remaining in the equilibrium with the supersaturated vapor. The total enthalpy is than equal to

$$
G_{\text {total }}=s M_{\mathrm{v}} g_{\mathrm{v}}+M_{1} g_{1}+M_{\mathrm{d}} g_{1}+4 \pi \gamma N r^{2} .
$$

Again, $G_{\text {total }}$ is conserved during the process so $\delta G_{\text {total }}=0$. The mass conservation law yields $\delta M_{1}=-\delta M_{\mathrm{d}}$. Finally we get $4 \pi \gamma \delta\left(N r^{2}\right)=-\delta s M_{\mathrm{v}} g_{\mathrm{v}}$. The changes of the supersaturation $\delta s$ yicld the changes of $N r^{2}$. When the process reaches equilibrium $s$ becomes constant and $\delta s=0$ which leads to

$$
N(r) \propto 1 / r^{2} .
$$

The stationary density of clusters decreases as their radius increases.

The considerations concerning enthalpy can only show the evidence of the coupling of $N, r^{2}$, and $s$ quantities, but does not enable us to find their co-dependent evolution. Let us try to postulate it. We know that $N r^{2}$ and $s$ are coupled, so their evolution should be treated as the evolution of two coupled physical quantities each 
of them dumped (for instance by diffusion). One of them has a source: supersaturation induced by the laser light. The simplest set of the differential equations of the first order describing such a situation takes the form

$$
\begin{aligned}
& \frac{\mathrm{d} x}{\mathrm{~d} t}=-a x+b x_{1}, \\
& \frac{\mathrm{d} x_{1}}{\mathrm{~d} t}=-c x_{1}-d x+e,
\end{aligned}
$$

where $\dot{x}$ and $x_{1}$ represent $N r^{2}$ and the supersaturation, respectively. The coefficients can be interpreted as follows: $a$ and $c$ describe the relaxation of $N r^{2}$ and the supersaturation, respectively, $b$ and $d$ are responsible for the coupling of these quantities and $e$ is the source term.

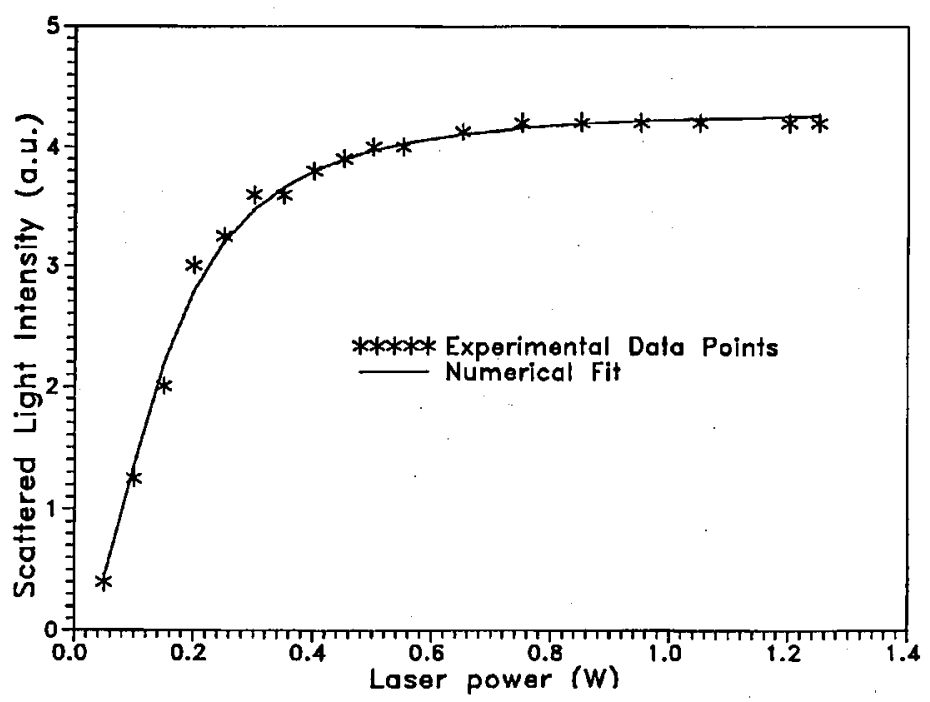

Fig. 4. The dependence of the scattered light intensity (He-Ne, $633 \mathrm{~nm}$ ) on the inducing light intensity.

We are interested in the evolution of $x=N r^{2}$

$$
\frac{\mathrm{d}^{2} x}{\mathrm{~d} t^{2}}+(a+c) \frac{\mathrm{d} x}{\mathrm{~d} t}+(b d+a c) x-b e=0 .
$$

The solution takes the form of the harmonic dumped oscillator

$$
x(t)=A \mathrm{e}^{-\Gamma t}[-\cos (\omega t)+C \sin (\omega t)]+1,
$$

where $\Gamma=(a+c) / 2, \quad \omega=\left[4 b d-(a-c)^{2}\right]^{1 / 2}$.

The initial conditions are

$$
\begin{array}{ll}
x(0)=0 & (\text { the clusters are absent) } \\
x_{1}(0)=s & \text { (initial supersaturation) }
\end{array}
$$

and

$$
x^{\prime}(0)=0, \quad x_{1}^{\prime}(0)=0 .
$$


It is worth noticing that for $t \rightarrow \infty, x \rightarrow x_{\infty}=b e /(a c+b d)$. So if $a, b, c, d$ and $e$ are linear functions of the light intensity $I$, the stationary value of the scattered light intensity would saturate square-like with $I$ (say: $I^{2} /\left(1+\alpha I^{2}\right)$ ). Such a dependence was observed in the experiment (Fig. 4).

\section{The scattering of light}

The process of the light scattering by metal spheres of any size in comparison to the light wavelength was described by Gustav Mie in 1908. Mie treated the interaction of spherical particles of arbitrary material with light by solving Maxwell's equations with appropriate boundary conditions.

The cross-sections $C$ describing those phenomena are calculated by integrating of the Poyinting vector averaged in time. Finally we get the cross-sections normalized to the area of the surface of cluster $Q=C / \pi r^{2}$, where $r$ is the radius of the droplet

$$
\begin{aligned}
& Q_{\mathrm{sca}}=2 / x^{2} \sum(2 n+1)(2 n+1)\left(\left|a_{n}\right|^{2}+\left|b_{n}\right|^{2}\right), \\
& Q_{\mathrm{ext}}=2 / x^{2} \sum(2 n+1)\left[\operatorname{Re}\left(a_{n}+b_{n}\right)\right], \\
& Q_{\mathrm{abs}}=Q_{\mathrm{ext}}-Q_{\mathrm{sca}},
\end{aligned}
$$

where $x=k r$ and $k=2 \pi / \lambda$ is the light wave vector. The functions $a_{n}$ and $b_{n}$ are given by the formulas

$$
\begin{aligned}
& a_{n}=\frac{\Psi_{n}(x) \Psi_{n}^{\prime}(m x)-m \Psi_{n}(m x) \Psi_{n}^{\prime}(x)}{\xi_{n}(x) \Psi_{n}^{\prime}(m x)-m \Psi_{n}(m x) \xi_{n}^{\prime}(x)} \\
& b_{n}=\frac{m \Psi_{n}(x) \Psi_{n}^{\prime}(m x)-\Psi_{n}(m x) \Psi_{n}^{\prime}(x)}{m \xi_{n}(x) \Psi_{n}^{\prime}(m x)-\Psi_{n}(m x) \xi_{n}^{\prime}(x)}
\end{aligned}
$$

where $\Psi_{n}(x)$ is the Ricatti-Bessel function of order $n$, and $\xi_{n}(x)$ is the Hankel function of the first kind of order $n$. The quantities $a_{n}$ and $b_{n}$ depend on the radius of particles $r$, on the light wavelength $\lambda$ and on the ratio of particle complex index of refraction to the refraction index of surrounding medium $m ; n$ is the order of the multipole series, $a_{n}$ and $b_{n}$ correspond to TM and TE modes, respectively.

In order to make any conclusions we have to compare the experimental results with the theoretical results. At the beginning as a first approximation it is best to have a relatively simple theoretical formula by means of which we can reconstruct the experimental dependencies qualitatively. It is difficult to use function series for this purpose. It is quite natural to assume that $x$ and $m x$ are small, i.e., the radius of the particle is small in comparison with the light wavelength both inside and outside the particle. The scattering takes place only on the induced electric dipole then. Such an approximation is known as the Rayleigh scattering. The scattering cross-section and the absorption in this case vary as the size of the particle to the sixth power and to the third power, respectively:

$$
C_{\mathrm{sca}} \propto k^{4} r^{6}, \quad C_{\mathrm{abs}} \propto k r^{3} .
$$


The experimental dependencies (Fig. 3) seem to be more complicated than the Rayleigh formulas simulation - the signal modulation that indicates the existence of the interference is worth noticing. The final average cluster size is about $1 \mu \mathrm{m}$, and it is why we see them as separated ob. . They are not small in comparison with the light wavelength that is about halt micron. The model proposed independently by Rayleigh and Guns [4] seems to be very suitable here. This model combines Rayleigh scattering and interference, i.e., each element of the volume of particle scatters the light independently as the electric dipole. The scattered electromagnetic field interferes, which results in the appearance of the train of minima and maxima depending on the size of the scatterer. The intensity of the light scattered by a single particle is

$$
i=k^{4} V^{2}|m-1|^{2} I_{0} G^{2}[2 x \sin (\theta / 2)]\left[1+\cos ^{2}(\theta)\right],
$$

where $x=k r=2 \pi r / \lambda, \theta$ is the scattering angle, and $G(u)=\left(9 \pi / 2 u^{3}\right)^{\frac{1}{2}} J_{\frac{3}{2}}(u)$. We approximate $J_{\frac{3}{2}}(u)$ functions with their asymptotic values: $J_{\frac{3}{2}}(u) \approx$ $a \cos [(u-\pi) / u]$. Finally we obtain a simple expression that illustrates the essential of the Rayleigh-Guns scattering for $\theta=90^{\circ}$. Each particle scallers proportionally to its surface area $\left(r^{2}\right)$ and creates the interference oscillations of the scattered light intensity

$$
i\left(r, \theta=90^{\circ}\right) \propto I_{0} r^{2} \cos ^{2}(\sqrt{2} k r) .
$$

The set of many scattering particles, i.e., the cloud, scatters as a sum of independent elements. The interference phenomena arising from adding the amplitudes of light waves coming from different objects never take place, in practice. So the total scattered light intensity takes the form of

$$
I=\sum N(r, t) i(r)
$$

where $N(r, t)$ is the number of clusters of the radius $r$ at the moment $t$ and $i(r)$ is the intensity of the light scattered by the cluster of radius $r$.

To get a function joining the intensity, time, number and the radius of clusters we assume as the first, and as we will see, as the best approximation that at every moment the cluster radius (at least the average) is well defined. This radius varies linearly with time. It means that the rate of growth of the volume of the droplet is proportional to the area of the surface of the droplet.

The total scattered light intensity is

$$
I \propto I_{0} N(r, t) r^{2}[1+D \cos (\Omega t)],
$$

where $\sqrt{2} k r=2 \Omega t$. The intensity of the scattered light observed at the right angle to the direction of the light propagation is proportional to the total clusters' surface $N r^{2}$ and oscillates due to the interference of the light scattered by various areas on the cluster. The oscillation frequency $\Omega$ defines the rate of growth of clusters. The stationary cluster radius can be determined as

$$
r=\sqrt{2} \Omega t / k \text {. }
$$

The depth of the interference pattern - the visibility of the fringes - is defined by the $D$ parameter. On the other hand the amplitude of the interference structure seen in the experiment decays with time (see, e.g. Fig. 3). So we must introduce 
not only the rate of growth (the frequency) but also its distribution (the frequency spectral width). The easiest way to do it is to assume that $D=d \exp (-\gamma t)$.

\section{Results}

Finally we obtain the following formula for the scattered light intensity (Eqs. (10), (23)):

$$
I(t)=A\left\{\mathrm{e}^{-\Gamma t}[-\cos (\omega t)+C \sin (\omega t)]+1\right\}[1+D \cos (\Omega t)],
$$

where the amplitudes $A, C$ and $d$, the decay constants $\Gamma$ and $\gamma$ and the frequencies $\Omega$ and $\omega$ are the parameters found by fitting the theoretical model expression (25) to the experimental data. The results are drawn as continuous lines in Figs. 2 and 3 .

The most interesting for us are the rates of growth of clusters $\Omega$. In Figs. 5, 6 and 7 the dependencies of the rate of growth upon the temperature of the cell $T$, the buffer gas pressure $p$ are shown. It can be seen that, in a wide range of $T$ and $p, \Omega$ is constant and equals about $0.015 \mu \mathrm{m}$. Only for high $T$ and $p$ it starts to grow.
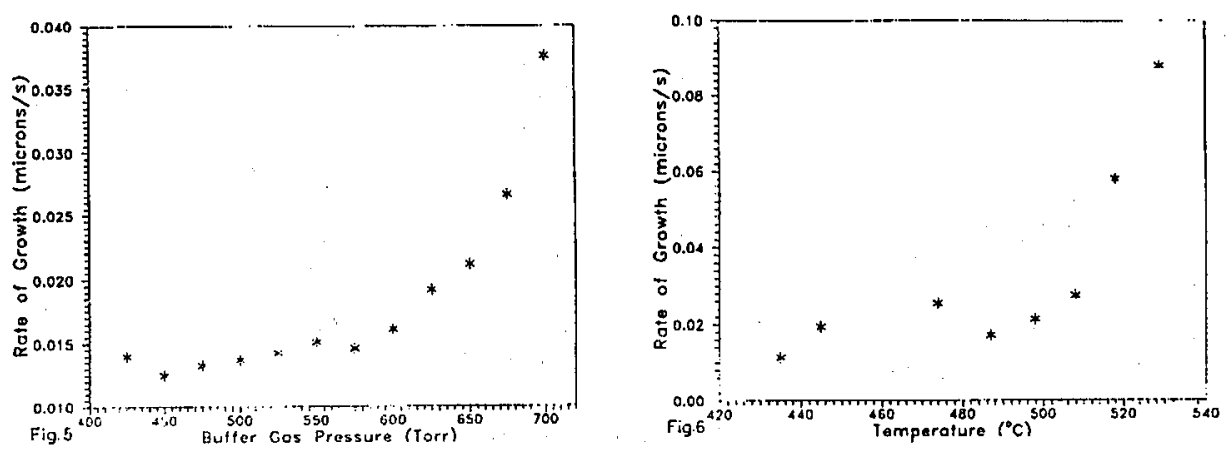

Fig. 5. The ratc of growth of cluster as a function of the buffer gas pressure.

Fig. 6. The rate of growth of cluster as a function of the temperature of the cell.

The dependencies upon the laser light intensity also secm to be very interesting. For modcrate pressure of about 500 torr $\Omega$ does not really depend on the inducing light intensity, but for higher pressures (600 torr) $\Omega$ is an increasing function of the laser power (Fig. 7). We get similar results for stationary clustcr size calculated by multiplying the evolution time by $\Omega$ (Figs. 8,9 and 10). Cluster radius behaves similarly to $\Omega$ as a function of the buffer gas pressure and the temperature of the cell. The dependence on laser power for the 600 torr pressure is different - the cluster radius decreases with laser power until it reaches $1.2 \mathrm{~W}$ and then slightly increases (Fig. 10). This dependence can be understood with the help of Eq. (3). Increasing laser power makes $s$ increasc and therefore $r$ decreases.

Under these conditions the clusters are bigger for smaller laser powers. The final increase in $r$ may be interpreted as caused by the increase in the temperature of the medium leading to the increase in the stable clusters radii. 

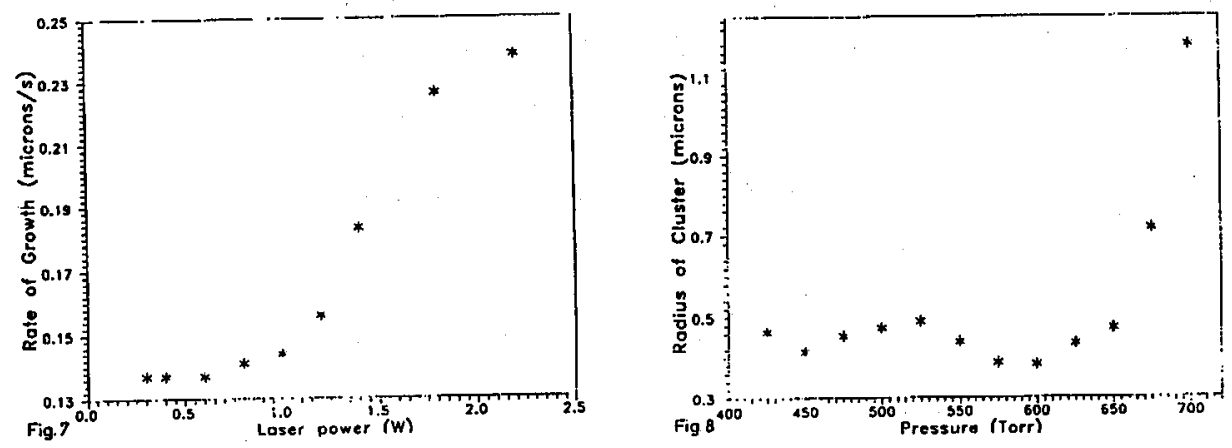

Fig. 7. The rate of growth of cluster as a function of the inducing light intensity. Fig. 8. The average radius of the cluster as a function of the buffer gas pressure.
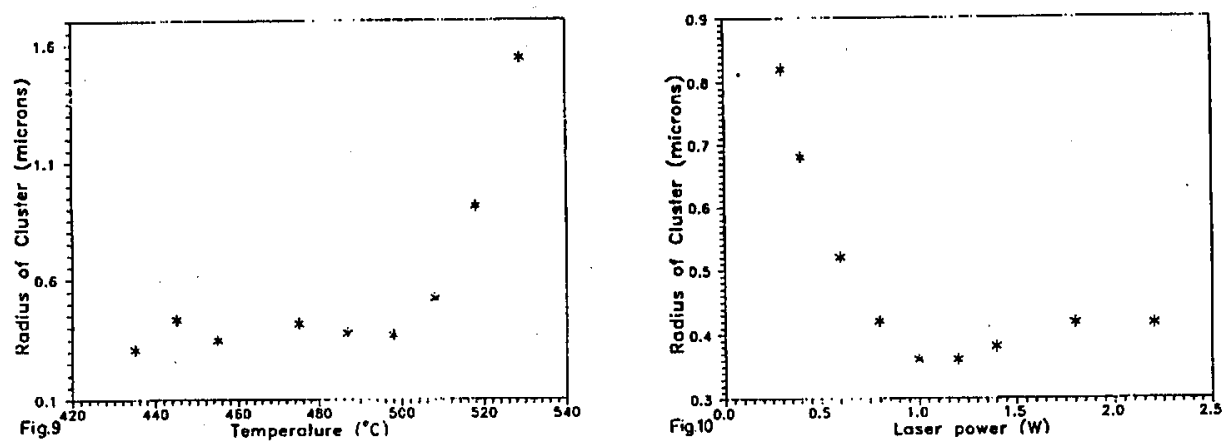

Fig. 9. The average radius of the cluster as a function of the temperature of the cell. Fig. 10. The average cluster radius as a function of the inducing light intensity.

\section{Acknowledgments}

This work was supported by the Committee for Scientific Research, grant No. 204859101.

\section{References}

[1] J. Kowalski, T. Stehlin, M. Volmer, Phase Transitions 24-26, 737 (1990).

[2] M. Allegrini, P. Biccli, D. Dattrino, L. Moi, Opt. Commun. 49, 29 (1984).

[3] K. Kolwas, M. Kolwas, P. Zalicki, Phys. Rev. Lett. A 167, 272 (1992); B. Inat, D. Jakubczyk, K. Kolwas, M. Kolwas, Acta Phys. Pol. A 81, 629 (1992).

[4] H.C. van de IIulst, Light Scaltering by Small Particles, Wiley, New York 1957.

[5] K. Huang, Statislical Mechanics, Wiley, New York 1963.

[6] A.N. Nesmieyanov, Vapor Pressure of the Chemical Elements, Elsevier, New York 1963. 